\title{
Gut microbiome, antibiotic use, and immunotherapy responsiveness in cancer
}

\author{
Jarred P. Reed, Suzanne Devkota, Robert A. Figlin \\ Cedars-Sinai Medical Center, Los Angeles, CA, USA \\ Correspondence to: Robert A. Figlin. Samuel Oschin Comprehensive Cancer Institute, Cedars-Sinai Medical Center, 8700 Beverly Blvd, Saperstein \\ Critical Care Tower, C 2003, Los Angeles, CA 90048, USA. Email: robert.figlin@cshs.org. \\ Provenance: This is an invited article commissioned by the Section Editor Dr. Xiao Li (Department of Urology, Jiangsu Cancer Hospital, Jiangsu \\ Institute of Cancer Research, Nanjing Medical University Affiliated Cancer Hospital, Nanjing, China). \\ Comment on: Tinsley N, Zhou C, Tan G, et al. Cumulative Antibiotic Use Significantly Decreases Efficacy of Checkpoint Inhibitors in Patients with \\ Advanced Cancer. Oncologist 2019. [Epub ahead of print].
}

Submitted Sep 27, 2019. Accepted for publication Oct 10, 2019.

doi: $10.21037 /$ atm.2019.10.27

View this article at: http://dx.doi.org/10.21037/atm.2019.10.27

Immune checkpoint inhibitors (ICIs) have transformed the treatment of solid malignancies, but responses are heterogeneous with benefit generally limited to only a fraction of patients. A number of factors have been hypothesized to contribute to variability in ICI efficacy. Among these, a growing body of evidence points to a critical role for the commensal gut microbiome, the complex ecosystem of microorganisms living together within the human gastrointestinal tract. Preclinical models studying the interplay between the gut microbiota and ICIs support the idea that the gut microbiome plays a critical role in immunotherapy responsiveness. For example, when germ-free mice were colonized with bacteria shown to be enriched in murine and human responders to ICIs, immune responsiveness was augmented via increased $\mathrm{T}$ helper 1 response, increased frequency of tumor-residing Batf3-lineage dendritic cells, and decreased frequency of colon-derived peripheral regulatory T-cells $(1,2)$. Chronic antibiotic therapy is known to lead to gut dysbiosis and may disrupt this association, potentially diminishing the benefit of ICIs. Recently, several groups have reported a negative correlation between antibiotic exposure and outcomes for patients receiving treatment with ICIs for advanced solid cancers (1,3-7). In this editorial, we discuss work by Tinsley et al., who found that cumulative antibiotic exposure diminishes ICI efficacy (8).

In this single-site, retrospective study, medical records from 291 patients [ $\mathrm{n}=179$ melanoma, $\mathrm{n}=64$ non-small cell lung cancer (NSCLC), and $\mathrm{n}=48$ renal cell carcinoma] undergoing ICI treatment were reviewed for antibiotic exposure occurring within the time period two weeks before and six weeks after initiation of ICI therapy. Ninetytwo patients $(32 \%)$ in the study received antibiotics. Univariate analyses were performed to assess for significant associations between patient characteristics and outcomes. Progression-free survival (PFS) was found to correlate with antibiotic exposure, performance status, and comorbidity. Overall survival (OS) correlated with each of these factors, along with clinical trial enrollment and presence of three or more metastatic sites. Multivariate analysis controlling for clinical factors with $\mathrm{P}<0.2$ on univariate analysis showed that antibiotic use was associated with significantly shorter PFS (HR 1.40; $\mathrm{P}=0.033$ ) and OS (HR 1.487; $\mathrm{P}=0.033$ ). An exploratory analysis was then performed to assess whether degree of antibiotic exposure had an effect on outcomes. Patients were grouped into three cohorts: no antibiotic treatment, a single course of antibiotics for 7 or fewer days, or "cumulative" treatment with multiple courses or treatment for more than 7 days. The authors found that while patients who received cumulative antibiotics had shorter PFS and OS, antibiotic exposure for 7 or fewer days had less effect and was associated with only a nonsignificant trend toward decreased PFS and OS. Median survival was 21.7 months in the group with no antibiotics, 17.7 months $(\mathrm{P}=0.294)$ in the group who received 7 days or less of antibiotics, and 6.3 months $(\mathrm{P}=0.009)$ in the group exposed to cumulative antibiotics. The authors assert that this is a novel and clinically important finding: cumulative 
antibiotic exposure is associated with diminished outcomes for patients receiving ICIs for advanced cancer (8).

It is important to place this report into the context of a growing body of studies which have incrementally extended our understanding of the association between antibiotic exposure and ICIs. For example, Ahmed et al. performed a retrospective cohort study of 60 patients who received ICIs and found that those exposed to antibiotics within 2 weeks before and/or after the first dose of immunotherapy had reduced response rates (RRs) and shorter PFS and OS. Interestingly, exposure to narrow-spectrum antibiotics, defined as only covering Gram-positive bacteria, did not appear to affect RR (3). Another retrospective cohort study from Derosa et al. of 170 patients treated with ICIs found that antibiotic exposure within 2 months prior to or 1-month after initiation of ICI treatment led to shorter PFS and OS. Notably, subgroup analysis showed that within this cohort antibiotic therapy was associated with shorter PFS for all cancer types (which included NSCLC, renal cell carcinoma, and urothelial carcinoma), but survival was decreased only in NSCLC (4). Sen et al. retrospectively analyzed 172 patients with a wide variety of solid malignancies being treated with ICIs within the context of phase I trials. While antibiotic use within the time period of 30 days prior to initiation of ICI treatment was associated with decreased OS, antibiotic exposure within 30 days or from 30-60 days after initiation of ICIs had no effect on PFS, OS, or primary progressive disease (5). As Tinsley and colleagues note, there have been two small retrospective studies which failed to show any association between antibiotic therapy and ICI efficacy; these cohorts were limited to patients with NSCLC treated specifically with nivolumab $(9,10)$.

Importantly, following publication of this report by Tinsley and colleagues the first prospective study in this space was reported by Pinato et al., who performed a multicenter, cohort trial of 196 patients with solid cancers treated with ICIs. These authors found that antibiotic therapy prior to ICI treatment was associated with worse OS (2 vs. 26 months, HR 7.4; 95\% CI, 4.3-12.8; $\mathrm{P}<0.001$ ) and a higher likelihood of primary refractoriness to ICI therapy [21 of 28 (81\%) vs. 66 of $151(44 \%) ; \mathrm{P}<0.001$ ]. Interestingly, these effects were not seen in patients who received antibiotics after initiation of ICI therapy (HR 0.9; $95 \%$ CI, 0.5-1.4, $\mathrm{P}=0.76$ ). The negative effect of prior antibiotic therapy held across all tumor types (groups included NSCLC, melanoma, and other) and in multivariate analysis was independent of tumor site, disease burden, and performance status (11).

Taken together, current evidence points to a striking interaction between antibiotic exposure and outcomes in patients treated with ICIs. Tinsley and colleagues extend this understanding by showing that within the context of immunotherapy, treatment with multiple or prolonged courses of antibiotics appear to be more harmful than isolated short exposures. As with most other work within this space, this study is limited by its retrospective nature, which may introduce bias in patient selection and analysis, and that patients came from a single center. Additionally, the authors did not assess the impact of timing of antibiotic therapy in relation to ICI treatment within the antibioticexposed cohort. Given prior reports suggest that antibiotic use prior to initiation of ICI therapy may be more deleterious than concurrent treatment, it would be useful to examine whether there is an interaction between timing of antibiotic therapy and cumulative exposure. Along these lines, the boundaries of what constitutes pre-treatment antibiotic exposure require better definition. While the authors offer a rationale for selecting their timepoint of 2 weeks prior to initiation of ICI treatment, the definition of antibiotic exposure in various studies is heterogeneous and largely arbitrary, and further assessment of this aspect should be a focus of future work.

Another limitation of the report from Tinsley et al. is the absence of correlative studies of the gut microbiota in their cohorts. Such work has been performed by other groups and furthered our understanding of the specific elements of the composition of the microbiome which may be critical to ICI responsiveness. For example, Gopalakrishnan and colleagues have shown that alpha diversity, the average species diversity within a given patient, correlates with antiPD-1 response in patients with melanoma (12). Previous clustering analyses of gut microbiome composition in human subjects have shown diverging patterns of composition of commensal gut flora between responders and non-responders in various tumor types. Examples of species whose enrichment appear to be important for immunotherapy responsiveness include Bifidobacterium longum, Collinsella aerofaciens, and Enteroccous faecium in patients with metastatic melanoma (1); Akkermansia muciniphila in lung and kidney cancer (6); and Ruminococcaccae species in melanoma (12). Evidence also exists for the importance of specific species in response to particular therapies, such as Bacteroides fragilis for antiCTLA-4 therapy or Bifidobacterium in maturing intratumoral dendritic cells in anti-PD-L1 therapy $(13,14)$.

How can we utilize the insights from Tinsley and others 
to benefit patients at present? The development of expert consensus guidelines for antibiotic use in patients receiving or potentially undergoing ICI therapy would be helpful to raise clinician awareness and provide a framework for appropriate use. While conventional chemotherapy suppresses the immune system and the management of infectious complications related to cancer treatment is among the most critical aspects of patient care in patients receiving these treatments, ICIs are not generally immunosuppressive and pose less infectious risk. Given burgeoning evidence that antibiotics may negatively impact the efficacy of ICIs, their use in this population should be more targeted and judicious. Based on available evidence, we would recommend that clinicians limit both the time course and spectrum of antibiotic therapy as much as possible in patients who are planning to initiate or currently undergoing ICI therapy. Examples of other potential recommendations include: avoiding or deferring initiation of ICI therapy in patients who have recently received or are currently receiving broad spectrum antibiotics; consideration of treatment options other than ICIs in patients with chronic or recurrent infections requiring antimicrobial treatment; and deferring elective procedures that would require the use of Gram-negative antimicrobial prophylaxis in patients receiving ICI therapy.

Beyond aiding our understanding the effect of antibiotics on the interplay between the gut and ICIs, the development of a more thorough and granular grasp of which gut bacteria are critical to the immune response will likely lead to broader applications that could allow us to harness the gut to our benefit. Examples include pre-treatment assessment of the gut microbial signature to predict likelihood of response and potentially guide therapeutic decisionmaking, or manipulation of the microbiome in patients with "unfavorable" gut profiles to augment treatment response. Such interventions have already shown promise in pre-clinical mouse models of solid cancers. Germ-free or antibiotic-treated mice, which have been shown to have impaired ICI responsiveness compared to controls, can experience restoration of ICI efficacy with interventions such as co-housing with immune responsive mice, microbial supplementation, or fecal microbiota transplantation from feces of mouse or human responders to ICIs $(1,12)$.

Our nascent but growing understanding of the interplay between the gut, the immune system, and cancer holds great promise for our patients. The significance of these interactions may extend far beyond the bounds of our current apprehension. In a striking example, a recent study of the gut microbiome of patients with pancreatic cancer showed that long-term survivors display higher tumor microbial biodiversity and immune activation than short-term survivors and identified an intra-tumoral microbiome signature highly predictive of long-term survival. Furthermore, it was shown that the gut microbiome modulates the pancreatic tumor microbial landscape, and that fecal microbial transplantation from short- and longterm pancreatic cancer survivors into mouse models had differential effects on tumor growth (15). As recently as a decade ago we had essentially no knowledge of the effects of the gut on the immune response to solid tumors. While it remains to be seen what other unexpected interactions may be uncovered, our advice at this point would be to stay tuned.

\section{Acknowledgments}

None.

\section{Footnote}

Conflicts of Interest: The authors have no conflicts of interest to declare.

Ethical Statement: The authors are accountable for all aspects of the work in ensuring that questions related to the accuracy or integrity of any part of the work are appropriately investigated and resolved.

\section{References}

1. Matson V, Fessler J, Bao R, et al. The commensal microbiome is associated with anti-PD-1 efficacy in metastatic melanoma patients. Science 2018;359:104-8.

2. Geva-Zatorsky N, Sefik E, Kua L, et al. Mining the Human Gut Microbiota for Immunomodulatory Organisms. Cell 2017;168:928-43.e11.

3. Ahmed J, Kumar A, Parikh K, et al. Use of broadspectrum antibiotics impacts outcome in patients treated with immune checkpoint inhibitors. Oncoimmunology 2018;7:e1507670.

4. Derosa L, Routy B, Kroemer G, et al. The intestinal microbiota determines the clinical efficacy of immune checkpoint blockers targeting PD-1/PD-L1. Oncoimmunology 2018;7:e1434468.

5. Sen S, Carmagnani Pestana R, Hess K, et al. Impact of antibiotic use on survival in patients with advanced cancers treated on immune checkpoint inhibitor phase I clinical 
trials. Ann Oncol 2018;29:2396-8.

6. Routy B, Le Chatelier E, Derosa L, et al. Gut microbiome influences efficacy of PD-1-based immunotherapy against epithelial tumors. Science 2018;359:91-7.

7. Huemer F, Rinnerthaler G, Westphal T, et al. Impact of antibiotic treatment on immune-checkpoint blockade efficacy in advanced non-squamous non-small cell lung cancer. Oncotarget 2018;9:16512-20.

8. Tinsley N, Zhou C, Tan G, et al. Cumulative Antibiotic Use Significantly Decreases Efficacy of Checkpoint Inhibitors in Patients with Advanced Cancer. Oncologist 2019. [Epub ahead of print].

9. Kaderbhai C, Richard C, Fumet JD, et al. Antibiotic Use Does Not Appear to Influence Response to Nivolumab. Anticancer Res 2017;37:3195-200.

10. Hakozaki T, Okuma Y, Omori M, et al. Impact of prior antibiotic use on the efficacy of nivolumab for non-small cell lung cancer. Oncol Lett 2019;17:2946-52.

Cite this article as: Reed JP, Devkota S, Figlin RA. Gut microbiome, antibiotic use, and immunotherapy responsiveness in cancer. Ann Transl Med 2019;7(Suppl 8):S309. doi: 10.21037/ atm.2019.10.27
11. Pinato DJ, Howlett S, Ottaviani D, et al. Association of Prior Antibiotic Treatment With Survival and Response to Immune Checkpoint Inhibitor Therapy in Patients With Cancer. JAMA Oncol 2019. [Epub ahead of print].

12. Gopalakrishnan V, Spencer CN, Nezi L, et al. Gut microbiome modulates response to anti-PD-1 immunotherapy in melanoma patients. Science 2018;359:97-103.

13. Vétizou M, Pitt JM, Daillère R, et al. Anticancer immunotherapy by CTLA-4 blockade relies on the gut microbiota. Science 2015;350:1079-84.

14. Sivan A, Corrales L, Hubert N, et al. Commensal Bifidobacterium promotes antitumor immunity and facilitates anti-PD-L1 efficacy. Science 2015;350:1084-9.

15. Riquelme E, Zhang Y, Zhang L, et al. Tumor Microbiome Diversity and Composition Influence Pancreatic Cancer Outcomes. Cell 2019;178:795-806.e12. 